\title{
Atherosclearosis plaque formation is related to EndMT in diabetic minipigs with coronary heart disease and the effect of Traditional Chinese medicine FTZ Prescription
}

\section{Lexun Wang}

Guangdong Pharmaceutical University

DongXing Zhang

Guangdong Pharmaceutical University

Wenjing Zhan

Guangdong Pharmaceutical University

Jianying Yin

Guangdong Pharmaceutical University

Ke Wang

Guangdong Pharmaceutical University

Hong Wang

Guangdong Pharmaceutical University

Lixia Song

Guangdong Pharmaceutical University

Zhanhui Gu

Guangdong Pharmaceutical University

Caijuan Guo

Guangdong Pharmaceutical University

Zhihuan Zeng

Guangdong Pharmaceutical University

Qin Zhong

Guangdong Pharmaceutical University

Weixuan Wang

Guangdong Pharmaceutical University

Weijian Bei ( $D$ 806362747@139.com )

Guangdong Pharmaceutical University https://orcid.org/0000-0002-5135-3274

\section{Xianglu Rong}

Guangdong Pharmaceutical University

Jiao Guo

Guangdong Pharmaceutical University 
Original investigation

Keywords: diabetes, coronary heart disease, Traditional Chinese medicine FTZ, endothelial-tomesenchymal transition, inflammation

Posted Date: November 15th, 2021

DOI: https://doi.org/10.21203/rs.3.rs-1031026/v1

License: (c) (1) This work is licensed under a Creative Commons Attribution 4.0 International License. Read Full License 


\section{Abstract}

Background: Coronary heart disease (CHD) caused by diabetes mellitus (DM) is the main cause of death in diabetic patients. The treatment of diabetes complicated with coronary heart disease (DM-CHD) is still a big challenge in the medical practice. Endothelial-mesenchymal transition (EndMT) has been considered as the key process in endothelial dysfunction in the atherosclerotic diseases, including DMCHD. The traditional Chinese prescription Fufang Zhenzhu Tiaozhi (FTZ) formula is used to treat diabetes and dyslipidemia. Recently, several studies have shown the therapeutic effect of FTZ in cardiovascular diseases. However, the mechanism of the protection on coronary atherosclerosis is still needed for further investigation.

Objective: To evaluate the efficacy of FTZ in preventing DM-CHD and explore the specific mechanism of FTZ based on EndMT.

Methods: DM-CHD minipigs model constructed by using high-fat/high-sucrose/high-cholesterol diet (HSFCD) combined with streptozotocin (STZ) and coronary balloon injury, then randomly divided into control (Ctrl), DM-CHD model (Mod), DM-CHD treated with FTZ (FTZ) or positive drug (metformin and atorvastatin, $\mathrm{M}+\mathrm{A}$ ). Twenty-two weeks after administration, the cardiac functions of pigs were detected by ultrasound and electrocardiography (ECG). The stenosis and plaque of the left anterior descending (LAD) coronary artery were assessed through angiography, intravascular ultrasound (IVUS), and optical coherence tomography (OCT). Pigs were sacrificed after all examinations and the tissues were collected for further testing. The serum levels of blood glucose and lipid, myocardial injury markers, and inflammatory factors were detected. The biomarkers of EndMT were observed. The potential targets and signaling pathways were analyzed. The human umbilical vein endothelial cell (HUVEC) was cultured for testing the effect of FTZ under high glucose $(\mathrm{HG})$.

Results: In the Mod group, the serum levels of FBG, TC, TG, HDL-C, and LDL-C and the myocardial injury markers (LDH, CTnT, CK-MB) were increased significantly. The ST segment and the height of the T wave of ECG were significantly elevated in the Mod group. The results of coronary angiography, OCT, and IVUS showed that there was an obvious narrow in the coronary artery of the Mod group, and the pathological changes were mainly intimal fibrosis. Furthermore, the levels of TGF- $\beta 1$ and a-SMA significantly increased while the levels of CD31 and VE-cadherin notably decreased in the Mod group, which showed EndMT characteristic. Besides, the inflammation and apoptosis pathways were markedly activated in the Mod group. After treatment with FTZ, the glucolipid metabolism and the myocardium injury were effectively improved in DM-CHD minipigs. FTZ treatment improved the function of the heart and alleviated coronary stenosis. The expression of a-SMA in the coronary artery was significantly decreased, and the expression of CD31 and VE-cadherin were increased in the coronary artery of the FTZ group minipigs. In addition, FTZ treatement inhibited the expressions of the inflammatory relative protein $\mathrm{p}$ $\mathrm{IkB} / \mathrm{p}-\mathrm{NF}-\mathrm{kB} / \mathrm{IL}-1 \beta$ and the apoptosis proteins Bax/cleave-Caspase- 3 in the coronary artery tissue. Additionally, FTZ ameliorated the injury effect and high migration activity of HUVECs caused by HG. 
Conclusions: Coronary atherosclearosis plaque formation is related to EndMT and FTZ protect heart partly via inhibiting coronary endothelial EndMT and regulating inflammation in DM-CHD pigs.

\section{Background}

Diabetes mellitus (DM) is a metabolic disorder caused by genetic and environmental factors. As the major type of diabetes ( $>90 \%$ ), type 2 Diabetes mellitus (T2DM) is public health threat with a significant increase in the last decade and is estimated to afflict over 400 million people worldwide [1]. The prevalence of diabetes has increased quickly these years. In China, the prevalence of total diabetes increased from $9.7 \%$ in 2007 to $11.2 \%$ in 2017 among adults [2]. Cardiovascular disease (CVD) is the most common cause of death and disability in diabetic patients [3]. Cardiovascular deaths account for $44 \%$ of death in T1DM patients and $52 \%$ of deaths in T2DM patients around the world [4]. Various clinical studies have demonstrated that the prevalence of CVD is higher in diabetics than in non-diabetic patients $[1,2,4-6]$. Moreover, the risk of CVD increases with the increase of fasting blood glucose level even if the blood sugar level is not diagnosed as diabetes mellitus [5]. Initial presentations of CVD in DM most commonly manifest as heart diseases (including heart failure, angina, and non-fatal myocardial infarction), mainly caused by coronary atherosclerosis [6].

Coronary atherosclerosis, a chronic inflammatory disease caused by chronic inflammation, lipid accumulation, and immune disorder in the wall of arteries, is characteristic of endothelial dysfunction and plaque formation [7]. The endothelial cells can secrete numerous bioactive factors to prevent the invasion of harmful substances into the arterial wall and act as a selective diffusion barrier between the blood and the other wall layers [8]. Current theories suggest that the dysfunction of endothelial cells is one of the initial steps in the development of atherosclerosis [8]. As the initial pathologic change of atherosclerosis, endothelial dysfunction can promote the invasion of harmful substances (such as oxidation of lipoproteins) into the arterial wall, the infiltration of inflammatory cells to vascular intima, platelet, and leukocyte adhesion, and thrombus formation [8, 9]. Endothelial-mesenchymal transition (EndMT) has been indicated as a key process in endothelial dysfunction which always occurs during atherosclerosis [10-12].

EndMT is a recently described biological process in which endothelial cells (EC) lose the cell-cell contact and cell polarity and acquire the spindle-shaped morphology and functions of mesenchymal cells (such as fibroblasts and smooth muscle cells), including enhanced migratory and proliferative capacity, secretion of extracellular matrix proteins, and expression of various leukocyte adhesion molecules [10, 13] During the EndMT process, the expression of endothelial markers such as CD31 and VE-cadherin is reduced, whereas the mesenchymal markers including alpha-smooth muscle actin (a-SMA), N-cadherin, and calponin are acquired [10,14]. Under the induce of high glucose and lipid in diabetes, EndMT ECs acquire a proinflammatory state and become more permeable, promoting the accumulation of leucocytes and lipids in the intima of the artery, culminating in foam cell formation and the formation of a fatty streak [10]. In addition, EndMT promotes plaque growth by increasing deposition of proatherogenic extracellular matrix (ECM), along with increased expression of adhesion molecules ICAM and VCAM, 
thereby promoting adherence of leukocytes to ECs [15], and also enhances atherosclerotic plaque rupture by altering collagen-MMPs balance [16]. Although the mechanisms of EMT have been extensively studied [10-12], there are no effective means to treat atherosclerosis by targeting the EndMT.

Traditional Chinese medicine (TCM) has unique advantages in the treatment of glycolipid metabolism disorders (GLMD) [17]. Fufang Zhenzhu Tiaozhi (FTZ) formula, a patentable traditional Chinese herbal prescription established by Professor Jiao Guo under the guidance of her theory "Tiao Gan Qi Shu Hua Zhuo" to treat GLMD, is constituted with Coptidis Rhizoma, Ligustri Lucidi Fructus, Salviae miltiorrhizae radix et Rhizoma, Cirsii japonici herba, Eucommiae Cortex, Citri sarcodactylis Fructus, Notoginseng Radix et Rhizoma, and Atractylodes macrocephala Rhizoma [17-19]. Recent studies have identified the main components and potential targets of FTZ and demonstrated its protective effects in treating diabetes, hyperlipidemia, non-alcoholic fatty liver disease (NAFLD), and aging-induced osteoporosis [18-20]. Previous studies have shown that FTZ could inhibit the development of atherosclerosis in the atherosclerosis model and exhibit the cardioprotective effect in the diabetic minipig model with coronary heart disease (DM-CHD) [21-24]. However, the effect and molecular mechanisms underlying FTZ in DM$\mathrm{CHD}$ are not clear.

Therefore, we established a DM-CHD model in Wuzhishan minipigs by using a high-fat/highsucrose/high-cholesterol diet (HFSCD) and balloon injury of the coronary artery. And this study aimed to investigate the cardioprotective effect of FTZ and understand the mechanism underlying the beneficial effects of this TCM in vivo and in vitro.

\section{Methods}

\section{Preparation of FTZ}

The preparation of FTZ extract was consistent with the protocol described previously [24].

\section{Animals}

20-22 weeks old castrated male Chinese Wuzhishan minipigs were purchased from Guangdong Laboratory Animals Monitoring Institute (Certification: SYXK Guangdong 2017-0125, Guangzhou, China). They were housed in a single pen under controlled conditions (temperature between $24^{\circ} \mathrm{C}$ and $26^{\circ} \mathrm{C}$, relative air humidity $30 \%-70 \%$ ). The pigs were fed twice a day with a total daily amount of $3 \%$ of bodyweight and filtered tap water was available randomly. The study was approved by the Guangdong Pharmaceutical University Ethics Committee on Animal Care (Approval N: GDPULAC2015090), and the methods were carried out following the Guide for the Care and Use of Laboratory Animals (2011).

\section{The induction of diabetic minipigs with coronary heart disease}

After 1 week of adaptive feeding, minipigs were randomly divided into two groups: the control group (Ctrl, $n=4)$, the diabetes mellitus group ( $D M, n=14)$. The DM group was induced by intravenous injection of 
streptozotocin (STZ) (Sigma-Aldrich, St. Louis, MO, USA) at a dose of $90 \mathrm{mg} / \mathrm{kg}$ body weight for the first day and a dose of $60 \mathrm{mg} / \mathrm{kg}$ body weight for the second day, and then fed with the high-fat, high-sucrose, and high-cholesterol diet (HFSCD, 1.5\% cholesterol, 35\% sugar, $10 \%$ lard oil, $5 \%$ egg yolk powder, and $48.5 \%$ commercial standard pellet diet) for 4 weeks. The Ctrl pigs were injected with the same volume of vehicle (0.01 M citrate buffer, $\mathrm{pH} 4.5$ ) and received a normal diet (ND).

After 4 weeks of ND or HFSCD feeding, the coronary artery disease minipigs were induced by balloon injury, and the detailed protocol of surgery was described previously [24].

\section{Animal treatment}

After the surgery, the DM pigs were randomly allocated into three groups: model group (Mod, $n=4)$, FTZ group (FTZ, $n=5)$, and positive control drug group (Metformin + Atorvastatin, M+A, $n=5) . F T Z(1.2 \mathrm{~g} / \mathrm{kg})$ was given with meals in the FTZ group daily [24]. Metformin $(57.08 \mathrm{mg} / \mathrm{kg} / \mathrm{d}$, according to the daily dose of Metformin in the clinical treatment of $2.550 \mathrm{~g} / 60 \mathrm{~kg})$ and Atorvastatin $(0.92 \mathrm{mg} / \mathrm{kg} / \mathrm{d}$, according to the daily dose in the clinical treatment of $40 \mathrm{mg} / 60 \mathrm{~kg}$ ) were given with meals in M+A group. The treatment duration was 22 weeks. During this period, the pigs of the Mod, FTZ, and M+A group were given HFSCD while the Ctrl group received ND. All animal blood samples were collected monthly using heparinized tubes. Animals were sacrificed at the end of the experiment. Blood and coronary artery tissue were sampled for further pathological analysis and biochemical markers determination.

\section{Angiography}

The animals were anesthetized with Zoletil 50 injection(Virbac,NicelFrance), which contains tiletamine, a separate anesthetic component, and zolazepam (with the effects of both sedative and muscle relaxation) $5 \mathrm{mg} / \mathrm{kg}$ intravenously. The analysis of imaging results of left anterior descending branch (LAD) was described as previously with CGO-2100 (Digital Subtraction Angiography system, Wandong Medical, Peking, China) [24]. The measurement indicators include: (1) the length of two different angles of the target vessel; (2) the sum of the proximal diameter and distal diameter of the lesion as the reference vascular diameter; (3) minimal lumen diameter rate $(M L D \%)=(1$ - actual diameter/reference diameter $) \times$ $100 \%$ (the average sum of the diameter stenosis rates of two different angles); (4) minimum lumen area rate $(M L A \%)=(1$ - actual stenosis area/reference area) $\times 100 \%$ (the average sum of the area narrow rates of two different angles) [25].

\section{Intravascular ultrasound (IVUS)}

Under general anesthesia of pigs, IVUS pullback images were generated with Atlantis SR Pro $40 \mathrm{MHz}$ catheters and iLab 220C27 Ultrasound Imaging system (Boston Scientific, Natick, Massachusetts). The protocol of IVUS was described previously $[26,27]$. At 0,20 weeks after the coronary angiography, 0.014 guide wire was inserted into each pig. 40-MHz ultrasound catheter was inserted into the middle and lower part of the LAD and iliac artery along the guide wire, respectively, and the IVUS catheter was withdrawn manually. Before the acquisition, heparin was administered intravenously. Intracoronary nitroglycerin was administered before every pull back. After positioning the IVUS catheter distal to the lesion of interest, an 
automated pullback was performed at $0.5 \mathrm{~mm} / \mathrm{s}$. When obvious lesions appeared, the severity and the length of the involved vessels were observed. The IVUS catheter was re-entered for image acquisition and recording. Each catheter was collected twice to obtain a good image effect for later analysis. At the same time, the IVUS probe position was determined by continuous X-ray fluoroscopy, and IVUS acquisition was triggered by continuous ECG. After the collection, the ultrasound catheter and guide wire were withdrawn successively, and continuous electrocardiogram (ECG) monitoring was performed during the operation. IVUS data is stored on hard disks and analyzed after experiments. Images were automatically analyzed with commercially available Imaging System software POLARIS 2.8 Intravascular ultrasound System Trace Assist ${ }^{\text {TM }}$. In-line Digital (ILD) Two-dimensional lumen diagrams are synthesized and displayed by the computer. The luminal, device and vessel areas were measured, and the neointimal and total plaque areas were calculated as previously described $[28,29]$.

\section{Optical coherence tomography (OCT)}

Under general anesthesia, OCT images were obtained with the C7-XR OCT imaging system (LightLab Imaging, Inc., St. Jude Medical, St. Paul, Minnesota). Motorized OCT pullbacks were performed at a rate of $20 \mathrm{~mm} / \mathrm{s}$. All images were acquired at 100 frames per second, displayed with a color look-up table, and digitally archived. Qualitative analyses were performed at 1-mm intervals with commercial software (ILUMIEN OPTIS, St. Jude Medical, MN). Cross-section lumen, device areas, and percent area stenosis were measured as previously described [28, 29]. Percentage area of stenosis was calculated as [1-(lumen area/inner area) $] \times 100[30,31]$.

\section{Electrocardiogram (ECG) test}

The 12-lead ECG was continuously recorded and followed by 5 min via using a 12 leads multichannel ECG recording system SD-700A (Shidi Medical Scientific Engineering Inc, Nanjing, JS, China), as previously described [24].

\section{Transmission electron microscopy (TEM)}

Transmission electron microscopy was performed to observe ultrastructural alterations of vascular endothelial cells in the heart. LAD tissues were fixed in $4 \%$ glutaraldehyde and then embedded in resin after dehydration. The sections with a thickness of $70 \mathrm{~nm}$ were cut using an ultramicrotome and stained with uranyl acetate and lead citrate. The results were observed with a JEM-1400 electron microscope (JEOL Ltd., Japan).

\section{Cell culture and treatment}

The human umbilical vein endothelial cell (HUVEC) was gifted from Doctor Qidong Liu (Tongji University) and routinely cultured in the recommended medium at $37^{\circ} \mathrm{C}$ in $5 \% \mathrm{CO} 2$ and $95 \%$ humidified atmosphere. HUVECs were cultured onto 6-well plates overnight and then treated with high glucose ( $\mathrm{HG}, 30 \mathrm{mM})$ and FTZ extracts at different concentrations $(5 \mu \mathrm{g} / \mathrm{ml}, 20 \mu \mathrm{g} / \mathrm{ml}$, and $50 \mu \mathrm{g} / \mathrm{ml})$ for $72 \mathrm{~h}$. The supernatant of cells was collected for further test.

\section{Cell scratch assay}


When the cells seeded in 6-well plate reached a confluent state, a single scratch was made using a sterile $200 \mu \mathrm{l}$ pipette tip and marked. The cells were then incubated with FBS-free culture medium alone or HG with/without containing different concentrations of FTZ. Images of the scratches were captured at 0,24 h with Olympus IX71 inverted microscope (Tokyo, Japan) at 100x magnification. The width of the scratch was analyzed using the Image pro plus software (Silver Springs, MD, USA).

\section{Western blot analysis}

Proteins were isolated from the ventricular homogenate or cells with lysis buffer (Beyotime Institute of Biotechnology, Shanghai, China) with PMSF (Sigma-Aldrich) and subjected to western blot as described previously [24]. The antibodies used in this study were: GAPDH, $\beta$-Tubulin, CD31, VE-Cadherin, a-SMA, Bcl2, Bax, IL-1 $\beta$, cleaved Caspase-3 (Abcam, Cambridge, MA, USA), p-IKB (p40, Ser32/36), p-NF-KB (p65, Ser536) (Cell Signaling Technology, Danvers, MA, USA).

\section{Cell counting kit-8 (CCK8) assay}

Cell viability was analyzed by CCK8 (Biosharp, Anhui, China) according to the manufacturer's protocol. Cells were seeded at a density of $5 \times 10^{3} /$ well into 96 -well microplates (Corning, USA) overnight. Then, the cells were treated with various concentrations of FTZ with or without HG. After treatment for $24 \mathrm{~h}$ or $48 \mathrm{~h}$, $10 \mu \mathrm{L}$ of CCK8 reagent was added to each well and then cultured for $1 \mathrm{~h}$. The absorbance was analyzed at $450 \mathrm{~nm}$ using a microplate reader (Molecular Devices, Silicon Valley, CA, USA). The proliferation of cells was expressed by absorbance.

\section{Enzyme-linked immunosorbent assay (ELISA) assay}

The pig serum levels of cardiac troponin T (cTnT), creatine kinase MB (CK-MB), interleukin $1 \beta$ (IL-1 $\beta$ ), IL18 , and transforming growth factor- $\beta 1$ (TGF- $\beta 1$ ) were tested by relative ELISA kits (Feiya Biotechnology, Jiangsu, China) according to the manufacturer's protocols. The levels of lactate dehydrogenase (LDH), IL$1 \beta$, and TGF- $\beta 1$ in cell supernatant were measured by LDH kit, IL-1 $\beta$ kit as well as TGF- $\beta 1$ kit (Feiya Biotechnology).

\section{Statistical analysis}

GraphPad Prism 8 (GraphPad Software Inc., La Jolla, CA, USA) was used for data analysis. Continuous variables were expressed as the means \pm SEM. ANOVA with Tukey's multiple comparisons test (equal variance) or the Kruskal-Wallis test, followed by Dunn`s Multiple Comparison test (unequal variance) was used for multiple comparisons. Two-sided tests were used throughout the study, and $p<0.05$ was considered statistically significant.

\section{Results}

\section{FTZ improved glucolipid metabolism in DM-CHD minipigs}


Our previous constructed the DM model by feeding with HFSCD alone and the pigs exhibited typical characteristics of T2DM after treatment for 12 weeks [24]. Due to a long time for inducing the model, we modified the protocol for the DM model in this study. DM model were induced by intraperitoneal injection of STZ for 2 consecutive days and fed with HFSCD simultaneously. After 4 weeks, the levels of serum total cholesterol (TC), low-density lipoprotein cholesterol (LDL-C), high-density lipoprotein cholesterol (HDL-C), and fasting blood glucose (FBG) were significantly increased in the DM group compared with that in the Ctrl group (Supplementary Fig. 1), similar to that induced by only HFSCD feeding 12 weeks [24].

After 4 weeks, all DM pigs were performed with balloon injury surgery and assigned to three groups: the model group (Mod), FTZ group (FTZ), and positive control drug group (Metformin + Atorvastatin, M+A). After treatment for 22 weeks, the levels of triacylglycerol (TG) and LDL-C in the FTZ group were significantly increased compared to those in the Mod group (Supplementary Fig. 1D, E). Although there was no statistical difference in the levels of FBG, HDL-C, and TC, their levels showed a reduced trend in the FTZ group compared with that in the Mod group (Supplementary Fig. 1A-C).

\section{FTZ alleviated the injury of the heart in DM-CHD minipigs}

The ECG results showed that compared with the Ctrl group, the ST segment and the height of the T wave were significantly elevated in the Mod group (Fig. 1A). The levels of cTnT and CK-MB notably increased in the Mod group compared to that in the Ctrl group (Fig. 1B). These results indicate that the DM-CHD model was successfully established by STZ + HFSCD combined with balloon injury in minipigs. After treatment with FTZ for 22 weeks, the ECG results showed that FTZ treatment reduced the elevation of the ST segment and $T$ wave in the DM-CHD model (Fig. 1A). Although there was no statistical difference in the ultrasonic indicators such as EF, FS, and IVS among groups, their levels showed an increasing trend in the FTZ group compared with that in the Mod group (Supplementary Fig. 2). In addition, the serum levels of CTnT and CK-MB significantly decreased in the FTZ group as well as $\mathrm{M}+\mathrm{A}$ group compared with that in the Mod group (Fig. 1B). These data indicate that FTZ treatment could alleviate the heart damage of DMCHD pigs.

\section{FTZ improved coronary stenosis in DM-CHD minipigs}

Next, we tested the stenosis of the coronary artery by using angiography, IVUS, and OCT. The results of the angiographic measurements showed that the MLD\% and MLA\% significantly increased in the Mod group, compared with that in the Ctrl group, while notably reduced after treatment with FTZ or $\mathrm{M}+\mathrm{A}$ (Fig. 2A), which suggested that FTZ as well as $M+A$ reduced the pathological intimal thickening of the injured coronary artery. The results of IVUS and OCT showed that the intimal thickening and coronary stenosis were found in the coronary artery of the Mod group, and FTZ treatment decreased the thickened intima and improved coronary stenosis in DM-CHD minipigs (Fig. 2B, C). 


\section{FTZ alleviated the inflammation in the coronary artery of DM-CHD minipigs}

Various studies have shown that inflammation plays a pivotal role in the occurrence and development of metabolic diseases $[24,32,33]$. We previously reported that the inflammatory cytokine levels of serum (TNF- $a$ and IL-6) and heart tissue significantly increased in DM-CHD minipigs [24]. In this study, we found that the serum levels of inflammatory cytokines IL-1 $\beta$ and IL-18 markedly increased in the Mod group compared with that in the Ctrl group (Fig. 3A). After FTZ administration for 22 weeks, the serum levels of IL-1 $\beta$ and IL-18 were significantly lower than those in the Mod group (Fig. 3A). Then, we tested the protein changes of IL-1 $\beta$ in the coronary artery by western blot. As shown in Fig. 3B, the protein level of IL-1 $\beta$ significantly increased in the Mod group compared to the Ctrl group, and the increased IL-1 $\beta$ was revised after FTZ or M + A treatment.

NF-KB signal pathway plays a critical role in the inflammatory response [34]. We assessed the phosphorylated state of $\mathrm{IKB}(\mathrm{p} 40)$ and the activity of NF-KB (p65) in the coronary artery. The protein levels of phosphorylated NF-KB and phosphorylated IKB were enriched in the coronary artery tissues of the Mod group while were significantly reduced in the FTZ group or M + A group (Fig. 3B).

\section{FTZ inhibited the apoptosis of the coronary artery in DM- CHD minipigs}

Due to the important role of apoptosis in the development of DM-CHD and coronary artery injury [35, 36], we assessed the changes of apoptosis in the coronary artery of minipigs. As shown in Fig. 4, the protein level of apoptotic markers Bax and significantly increased and the level of anti-apoptotic protein Bcl-2 also increased in the Mod group compared with that in the Ctrl group. Additionally, the cleave-Caspase 3 is enriched in the coronary artery tissues of the Mod group. These results indicate that enhanced apoptosis involves in coronary artery injury in DM-CHD minipigs.

After treatment with FTZ or $\mathrm{M}+\mathrm{A}$, the protein levels of Bax and cleave-Caspase3 were significantly reduced in the coronary artery tissue of DM-CHD minipigs (Fig. 4A). Unlike the reduced Bcl-2 in the $M+A$ group, its level notably increased in the FTZ group compared with that in the Mod group (Fig. 4A). Furthermore, the ratio of $\mathrm{Bcl}-2$ / Bax significant was increased in the FTZ group compared to in the Mod group (Fig. 4B). These data indicate that FTZ alleviated injury of the coronary artery in DM-CHD partly by inhibiting apoptosis.

\section{FTZ inhibited the coronary EndMT in DM-CHD minipigs}

We found that the serum level of TGF- $\beta 1$ significantly increased in the Mod group compared to the Ctrl group, and FTZ treatment decreased its level in DM-CHD pigs (Supplementary Fig. 3A). TGF- $\beta 1$ signal pathway plays an important role in the process of EndMT $[37,38]$. We tested the markers of endothelial cells and mesenchymal cells in the tissue of the coronary artery. As shown in Fig. 5A-D, compared within the Ctrl group, the protein level of a-SMA (the marker of mesenchymal cells) significantly increased while 
the levels of CD31, as well as VE-cadherin (the markers of endothelial cells), notably decreased in the Mod group, and the results of vascular endothelium ultrastructure by TEM showed obvious deposition of collagen fibers, indicating that EndMT has occurred in the coronary artery of DM-CHD pigs. Treatment with FTZ for 22 weeks, the level of a-SMA decreased markedly, the levels of CD31 and VE-cadherin increased, and the deposition of collagen fibers was improved in vascular endothelium (Fig. 5A-D). These data indicate that FTZ treatment improved the DM-CHD at least partly by inhibiting the EndMT of endothelial cells in the coronary artery.

\section{FTZ ameliorated the effect of high glucose on HUVECs}

Next, we assessed the protective effect of FTZ in vitro. First, the effect of FTZ on HUVECs was tested by CCK8. After treatment for $24 \mathrm{~h}, \mathrm{FTZ}$ has no obvious effect on HUVECs among 5-200 $\mu \mathrm{g} / \mathrm{ml}$ concentration (Supplementary Fig. 3B). Then we chose $5 \mu \mathrm{g} / \mathrm{ml}, 20 \mu \mathrm{g} / \mathrm{ml}$, and $50 \mu \mathrm{g} / \mathrm{ml}$ for further study. After treatment with high glucose $(\mathrm{HG}, 30 \mathrm{mM})$ for $48 \mathrm{~h}$, the cell viability was significantly reduced compared with normal glucose (Fig. $6 \mathrm{~A}$ ). $5 \mu \mathrm{g} / \mathrm{ml}$ and $50 \mu \mathrm{g} / \mathrm{ml} \mathrm{FTZ} \mathrm{treatment} \mathrm{revised} \mathrm{this} \mathrm{reduced} \mathrm{cell} \mathrm{viability}$ caused by $\mathrm{HG}$ after treatment for $48 \mathrm{~h}$, not $24 \mathrm{~h}$ (Fig. 6A, Supplementary Fig. 3C).

We tested the LDH of cell supernatant by ELISA. As shown in Fig. 6B, HG treatment notably increased the supernatant level of LDH and FTZ markedly inhibited the increase of LDH induced by HG. Furthermore, this inhibition of FTZ was dose-dependent (Fig. 6B). In addition, the supernatant levels of TGF- $\beta 1$ and IL$1 \beta$ were significantly increased in the $H G$ environment while markedly decreased under FTZ treatment in a dose-dependent way (Fig. 6C, D). These data indicate that FTZ improves the injury and the inflammation of HUVECs caused by high glucose.

The high migration activity is one characteristic of EndMT cells [11]. Next, we tested the migration activity of HUVECs through cell scratch test. As shown in Fig. 6E, HG significantly increased the migration activity of HUVECs, indicating that EndMT has occurred in HUVECs under HG. FTZ markedly inhbited the high migration activity of HUVECs caused by HG in a dose-dependent way (Fig. 6E).

\section{Discussion}

DM has become one of the most prevalent diseases in the last few decades and is now the seventh leading cause of death in the world [6]. Mortality in diabetes patients is mainly driven by the macrovascular complications of $\mathrm{CHD}$ and stroke [3]. As the leading cause of DM-CHD, atherosclerosis, especially coronary atherosclerosis causes plaque formation, arterial lumen stenosis, blood flow obstruction, and heart ischemia. The treatment of early atherosclerosis can not only advance the prevention of a series of fatal cardiovascular diseases but also greatly reduce the mortality and high cost of treatment of diabetic patients with CHD. In this study, we evaluated the anti-atherosclerosis effect of FTZ in the DM-CHD minipig model established by the combination of HFSCD and balloon injury. Our findings showed that FTZ treatment decreased the serum levels of TG and LDL-C, and improved the myocardial injury in the DM-CHD model. 
Coronary atherosclerosis, a long-lasting and continuously evolving disease, can cause multiple clinical manifestations, including asymptomatic and stable angina, acute coronary syndrome, heart failure, and sudden cardiac death [7]. As one of the characteristics of coronary atherosclerosis, the formation of plaque results in the stenosis of the coronary artery and insufficient blood supply to the heart [39]. In this study, the results of angiographic measurement, IVUS, and OCT showed the coronary stenosis in DM-CHD minipigs, indicating that the DM-CHD model was successfully established in minipigs. Moreover, FTZ improved the coronary stenosis in the heart of DM-CHD minipigs.

EndMT is an intricate cellular differentiation process whereby endothelial cells detach and migrate away from the endothelium and, to varying extents, decrease endothelial properties and acquire mesenchymal features [40]. As a typical kind of vascular endothelial injury, EndMT is considered as the initiation in atherosclerosis [10]. TGF $\beta$, inflammation, and altered shear stress have been identified as common inducers of EndMT in vascular endothelium [40]. Moreover, TGF- $\beta$ has been identified as the central player in driving EndMT progression $[15,40]$. In our study, we found that the serum level of TGF- $\beta 1$ increased in the Mod group, indicating that increased TGF- $\beta 1$ may promote the EndMT of vascular endothelial cells in the coronary artery. Besides, the levels of various proteins have changed in the EndMT process, such as the level of endothelial markers CD31 and VE-cadherin are reduced while the level of mesenchymal markers a-SMA is increased [11]. We found that the level of a-SMA increased while the levels of CD31 and VE-cadherin decreased in the coronary artery tissue of Mod group minipigs, indicating that there is the EndMT in the coronary endothelium of DM-CHD minipigs. Furthermore, FTZ significantly reduced the expression of a-SMA and increased the protein levels of CD31 and VE-cadherin in coronary artery tissue of DM-CHD minipigs. In addition, FTZ significantly inhibited the high migration activity of HUVECs caused by HG in vitro. These results indicate that FTZ alleviates coronary stenosis and reduces plaque formation at least partly by inhibiting the EndMT of vascular endothelial cells.

It is now accepted that inflammation plays a major role in all stages of coronary atherogenesis $[7,39,41$, 42]. Besides, inflammation also participates in the local, myocardial, and systemic complications of atherosclerosis [39]. The levels of IL-1 $\beta$ and IL-18 are increased in CHD patients and patients with advanced atherosclerosis $[43,44]$. Moreover, as the key mediators in the inflammatory cascade, IL- $1 \beta$ and IL-18 can drive plaque progression and instability [45]. In line with these studies, our data showed that the serum levels of IL-1 $\beta$ and IL-18 increased in the Mod group, and FTZ treatment significantly reduced the increase of these inflammatory factors. In addition, NF-KB is the pivotal transcription factor for regulating the proinflammatory cytokines. Under the proinflammatory stimulation, the $\mathrm{I} \mathrm{KB}$ is phosphorylated to release NF-kB, resulting in its activation, which promotes inflammation and is an important contributor to cell death in atherosclerosis [46-48]. In this study, we found that the phosphorylation levels of IKB and NF-KB increased in the coronary artery of DM-CHD minipigs. And FTZ treatment significantly reduced the release of inflammatory cytokines and inhibited the activation of the NF-KB pathway in the coronary artery. Combined with our previous research $[24,49]$, these data indicate that inflammation is a crucial factor in diabetes-related heart diseases including DM-CHD and diabetic cardiomyopathy, and FTZ may improve these heart diseases by inhibiting the inflammation. 
TCM has been widely used for thousands of years in China and in other Asian countries for the treatment of atherosclerotic diseases and cardiovascular diseases [50]. In the recent decade, various TCM formulae including FTZ have the hypolipidemic effect and anti-atherosclerosis in clinical observations and animal experiments [24, 50-52]. Our previous studies have confirmed that FTZ has protective effects in hyperlipidemia, diabetes, and NAFLD through regulating HMG-CoA Reductase (HMGCR) and cholesterol 7-alpha hydroxylase (CYP7A1), attenuating insulin resistance, and inhibiting the formation and activation of nucleotide-binding oligomerization domain-like receptor protein 3 (NLRP3) inflammasome, respectively $[18,19,24,53]$. In the cardiovascular aspect, FTZ has the cardioprotective effect in the treatment of DMCHD and diabetic cardiomyopathy (DCM) through inhibiting ERK and STAT3 signal pathways and activating PI3K/AKT pathway $[24,49]$. Furthermore, recent studies have shown that FTZ reduced restenosis in the rabbit restenosis model by regulating the adiponectin pathway and reducing NF-KB activity [21, 22], but the effect of FTZ on vascular endothelium has not been explicit. In this study, we found that FTZ significantly inhibited the EndMT of coronary artery in DM-CHD minipigs and revised the reduced cell viability of HUVECs caused by HG. These results indicate that FTZ might treat atherosclerosis by suppressing the EndMT of coronary vascular endothelium.

\section{Conclusions}

we found that coronary atherosclearosis plaque formation is related to EndMT in DM-CHD mihipigs. FTZ reduced hyperlipidemia, improved coronary stenosis, and attenuated inflammation and apoptosis of the coronary artery. Furthermore, FTZ treatment inhibited the EndMT. Thus, FTZ has therapeutic potential in the treatment and/or prevention of DM-CHD. Next, a clinical trial is needed to furtherly assess the effect of FTZ in DM-CHD patients.

\section{Abbreviations}

a-SMA: a-smooth muscle actin; Bax: Bcl-2-associated X; Bcl-2: B-cell lymphoma-2; CCK-8: Cell counting Kit-8; CHD: Coronary heart disease; CK-MB: Creatinine kinase-MB isoenzyme; cTn-T: Cardiac troponin-T; CVD: Cardiovascular disease; DM: Diabetes mellitus; DM-CHD: Diabetes complicated with coronary heart disease; ECG: Electrocardiography; ELISA: Enzyme-linked immunosorbent assay; EndMT: Endothelial to mesenchymal transition; FBG: Fasting blood glucose; GLMD: Glycolipid metabolism disorders; HDL-C: High density lipoprotein cholesterol; HSFCD: High-fat/high-sucrose/high-cholesterol diet; HUVEC: Human umbilical vein endothelial cell; IL-1ß: Interleukin-1B; IL-18: Interleukin-18; IKB: Inhibitor of NF-Kb; LAD: left anterior descending; LDH: Lactate dehydrogenase; LDL-C: Low density lipoprotein cholesterol; MLA: Minimum lumen are; MLD: Minimal lumen diameter; MMPs: Matrix metalloproteases; NAFLD: Nonalcoholic fatty liver disease; ND: normal diet; NF-кB: Nuclear factor kB; STZ: Streptozotocin; TC: Total cholesterol; TCM: Traditional Chinese medicine; TGF- $\beta$ : Transforming growth factor- $\beta 1$.

\section{Declarations}

\section{Ethics approval and consent to participate}


The study was approved by the Guangdong Pharmaceutical University Ethics Committee on Animal Care (Approval N: GDPULAC2015090), and the methods were carried out following the Guide for the Care and Use of Laboratory Animals (2011).

\section{Consent for publication}

If the manuscript is accepted, we approve it for publication in Cardiovascular Diabetology.

\section{Availability of data and materials}

The datasets supporting the conclusions of this article are included within the article.

\section{Competing interests}

The authors declare that they have no Competing interests.

\section{Funding}

This work was supported by the National Key Research and Development Program of China (2018YFC1704200), the Major basic and applied basic research projects of Guangdong Province of China (2019B030302005), the Key Project of the National Natural Science Foundation of China (81530102), the Basic and applied basic research project of Guangdong Province of China (2018A030313391, 2019A1515110123, 2020A1515010155, 2021A1515012553), and the "Innovation and Strengthening University Project" Subsidized Project of Guangdong Pharmaceutical University (2018KTSCX112). The funders had no role in study design, data collection, and analysis, manuscript preparation, or decision to publish.

\section{Authors' contributions}

Jiao Guo contributed to the conception and design of the study. Lexun Wang, Dongxing Zhang, Wenjing Zhan, Jianying Yin, and Weijian Bei contributed to the experiments, data analysis, and manuscript writing. Ke Wang, Hong Wang, Lixia Song, Zhanhui Gu, and Caijuan Guo contributed to the experiments and the data collection. Zhihuan Zeng contributed to the detection of heart function of minipigs. Weijian Bei and Xianglu Rong contributed to the tissue section analysis. Weixuan Wang, Weijian Bei and Xianglu Rong helped to revise the manuscript. All the authors have read and approved the final manuscript.

\section{Acknowledgements}

The 1st Affilated Hospital of Guangdong Pharmaceutical University, Mr. Jun Li, Mr. Yilian Zhou, and Mr. Zhongtao Wang; Shenzhen Advanced Medical Services Co. Ltd, Mr. Jiawen Lin, Mr. Geqi Wang, Mr. Yuan Hou, Mr. Hua Tang, and Mr. Yunqiang Tang supplied their excellent technology in Angiography, ECG, OCT, IVUS detection of the minipigs. We thank Dr Renjie Chi and Zehong Huang for technical assistance in the 
IVUS and OCT data Analysis. Mr. Jianfong Yu, Yijian Huang, Zhida Cai, Yadong Zhu, Zhifong Zhou, Ms Wenyi Liang, Yuping Li, Wei Xu and all have supplied their help in the experiment.

\section{Authors' information}

Guangdong Metabolic Diseases Research Center of Integrated Chinese and Western Medicine; Key Laboratory of Glucolipid Metabolic Disorder, Ministry of Education of China; Guangdong TCM Key Laboratory for Metabolic Diseases; Key Unit of Modulating Liver to Treat Hyperlipemia SATCM (State Administration of Traditional Chinese Medicine), SATCM Level 3 Lab of Lipid Metabolism; Institute of Chinese Medicine, Guangdong Pharmaceutical University, Guangzhou Higher Education Mega Center, Guangzhou, 510006, China.

\section{References}

1. Zimmet P, Alberti KG, Magliano DJ, Bennett PH: Diabetes mellitus statistics on prevalence and mortality: facts and fallacies. Nat Rev Endocrino/ 2016, 12(10):616-622.

2. Li Y, Teng D, Shi X, Qin G, Qin Y, Quan H, Shi B, Sun H, Ba J, Chen B et al: Prevalence of diabetes recorded in mainland China using 2018 diagnostic criteria from the American Diabetes Association: national cross sectional study. BMJ 2020, 369:m997.

3. Goodarzi MO, Rotter Jl: Genetics Insights in the Relationship Between Type 2 Diabetes and Coronary Heart Disease. Circ Res 2020, 126(11):1526-1548.

4. Morrish NJ, Wang SL, Stevens LK, Fuller JH, Keen H: Mortality and causes of death in the WHO Multinational Study of Vascular Disease in Diabetes. Diabetologia 2001, 44 Suppl 2:S14-21.

5. Henning RJ: Type-2 diabetes mellitus and cardiovascular disease. Future Cardio/ 2018, 14(6):491509.

6. Glovaci D, Fan W, Wong ND: Epidemiology of Diabetes Mellitus and Cardiovascular Disease. Curr Cardiol Rep 2019, 21(4):21.

7. Boudoulas KD, Triposciadis F, Geleris P, Boudoulas H: Coronary Atherosclerosis: Pathophysiologic Basis for Diagnosis and Management. Prog Cardiovasc Dis 2016, 58(6):676-692.

8. Milutinovic A, Suput D, Zorc-Pleskovic R: Pathogenesis of atherosclerosis in the tunica intima, media, and adventitia of coronary arteries: An updated review. Bosn J Basic Med Sci 2020, 20(1):21-30.

9. Tousoulis D, Simopoulou C, Papageorgiou N, Oikonomou E, Hatzis G, Siasos G, Tsiamis E, Stefanadis C: Endothelial dysfunction in conduit arteries and in microcirculation. Novel therapeutic approaches. Pharmacol Ther 2014, 144(3):253-267. 
10. Kovacic JC, Dimmeler S, Harvey RP, Finkel T, Aikawa E, Krenning G, Baker AH: Endothelial to Mesenchymal Transition in Cardiovascular Disease: JACC State-of-the-Art Review. J Am Coll Cardiol 2019, 73(2):190-209.

11. Souilhol C, Harmsen MC, Evans PC, Krenning G: Endothelial-mesenchymal transition in atherosclerosis. Cardiovasc Res 2018, 114(4):565-577.

12. Islam S, Bostrom KI, Di Carlo D, Simmons CA, Tintut Y, Yao Y, Hsu JJ: The Mechanobiology of Endothelial-to-Mesenchymal Transition in Cardiovascular Disease. Front Physiol 2021, 12:734215.

13. Nakajima Y, Yamagishi T, Hokari S, Nakamura H: Mechanisms involved in valvuloseptal endocardial cushion formation in early cardiogenesis: roles of transforming growth factor (TGF)-beta and bone morphogenetic protein (BMP). Anat Rec 2000, 258(2):119-127.

14. Chen PY, Schwartz MA, Simons M: Endothelial-to-Mesenchymal Transition, Vascular Inflammation, and Atherosclerosis. Front Cardiovasc Med 2020, 7:53.

15. Chen PY, Qin L, Baeyens N, Li G, Afolabi T, Budatha M, Tellides G, Schwartz MA, Simons M: Endothelial-to-mesenchymal transition drives atherosclerosis progression. J Clin Invest 2015, 125(12):4514-4528.

16. Evrard SM, Lecce L, Michelis KC, Nomura-Kitabayashi A, Pandey G, Purushothaman KR, d'Escamard V, Li JR, Hadri L, Fujitani K et al: Endothelial to mesenchymal transition is common in atherosclerotic lesions and is associated with plaque instability. Nat Commun 2016, 7:11853.

17. Guo J: Research progress on prevention and treatment of glucolipid metabolic disease with integrated traditional Chinese and Western medicine. Chin J Integr Med 2017, 23(6):403-409.

18. Hu X, Wang M, Bei W, Han Z, Guo J: The Chinese herbal medicine FTZ attenuates insulin resistance via IRS1 and PI3K in vitro and in rats with metabolic syndrome. $J$ Trans/ Med 2014, 12:47.

19. Guo J, Bei W, Hu Y, Tang C, He W, Liu X, Huang L, Cao Y, Hu X, Zhong X et al: A new TCM formula FTZ lowers serum cholesterol by regulating HMG-CoA reductase and CYP7A1 in hyperlipidemic rats. $J$ Ethnopharmacol 2011, 135(2):299-307.

20. Luo D, Li J, Chen K, Rong X, Guo J: Untargeted Metabolomics Reveals the Protective Effect of Fufang Zhenshu Tiaozhi (FTZ) on Aging-Induced Osteoporosis in Mice. Front Pharmacol 2018, 9:1483.

21. Li T, Zhang R, Liu Y, Yao Y, Guo J, Zeng Z: Fufang-Zhenzhu-Tiaozhi capsule ameliorates rabbit's iliac artery restenosis by regulating adiponectin signaling pathway. Biomed Pharmacother 2020, 128:110311.

22. Zhang R, Li T, Guo J, Zhao Y, Liu Y, Yao Y, Zeng Z: Fufang-Zhenzhu-Tiaozhi Capsule reduces restenosis via the downregulation of NF-kappaB and inflammatory factors in rabbits. Lipids Health 
Dis 2018, 17(1):272.

23. Tang CP, Jiang T, Guo J, Wei YP, Yang CY, Chen FC: [The effect of fufang zhenzhu tiaozhi capsule on lipid metabolism in atherosclerotic rabbits]. Zhong Yao Cai 2010, 33(8):1285-1289.

24. Song L, Zhang D, Guo C, Gu Z, Wang L, Yao YS, Wang H, Zeng Z, Wang W, Yang Y et al: The traditional Chinese medicine formula Fufang-Zhenzhu-Tiaozhi protects myocardia from injury in diabetic minipigs with coronary heart disease. Biomed Pharmacother 2021, 137:111343.

25. Thim T, Hagensen MK, Drouet L, Bal Dit Sollier C, Bonneau M, Granada JF, Nielsen LB, Paaske WP, Botker HE, Falk E: Familial hypercholesterolaemic downsized pig with human-like coronary atherosclerosis: a model for preclinical studies. Eurolntervention 2010, 6(2):261-268.

26. Thim T, Hagensen MK, Wallace-Bradley D, Granada JF, Kaluza GL, Drouet L, Paaske WP, Botker HE, Falk E: Unreliable assessment of necrotic core by virtual histology intravascular ultrasound in porcine coronary artery disease. Circ Cardiovasc Imaging 2010, 3(4):384-391.

27. Patel D, Hamamdzic D, Llano R, Patel D, Cheng L, Fenning RS, Bannan K, Wilensky RL: Subsequent development of fibroatheromas with inflamed fibrous caps can be predicted by intracoronary near infrared spectroscopy. Arterioscler Thromb Vasc Biol 2013, 33(2):347-353.

28. Cheng Y, Ferrone M, Wang Q, Perkins LEL, McGregor J, Redfors B, Zhou Z, Rapoza R, Conditt GB, Finn A et al: Impact of Coronary Atherosclerosis on Bioresorbable Vascular Scaffold Resorption and Vessel Wall Integration. JACC Basic Trans/ Sci 2020, 5(6):619-629.

29. Slottow TL, Pakala R, Okabe T, Hellinga D, Lovec RJ, Tio FO, Bui AB, Waksman R: Optical coherence tomography and intravascular ultrasound imaging of bioabsorbable magnesium stent degradation in porcine coronary arteries. Cardiovasc Revasc Med 2008, 9(4):248-254.

30. Gasior P, Cheng Y, Valencia AF, McGregor J, Conditt GB, Kaluza GL, Granada JF: Impact of Fluoropolymer-Based Paclitaxel Delivery on Neointimal Proliferation and Vascular Healing: A Comparative Peripheral Drug-Eluting Stent Study in the Familial Hypercholesterolemic Swine Model of Femoral Restenosis. Circ Cardiovasc Interv 2017, 10(5).

31. Koskinas KC, Feldman CL, Chatzizisis YS, Coskun AU, Jonas M, Maynard C, Baker AB, Papafaklis $\mathrm{MI}$, Edelman ER, Stone PH: Natural history of experimental coronary atherosclerosis and vascular remodeling in relation to endothelial shear stress: a serial, in vivo intravascular ultrasound study. Circulation 2010, 121(19):2092-2101.

32. Hotamisligil GS: Inflammation and metabolic disorders. Nature 2006, 444(7121):860-867.

33. Franceschi C, Garagnani P, Parini P, Giuliani C, Santoro A: Inflammaging: a new immune-metabolic viewpoint for age-related diseases. Nat Rev Endocrinol 2018, 14(10):576-590. 
34. Kunnumakkara AB, Shabnam B, Girisa S, Harsha C, Banik K, Devi TB, Choudhury R, Sahu H, Parama D, Sailo BL et al: Inflammation, NF-kappaB, and Chronic Diseases: How are They Linked? Crit Rev Immunol 2020, 40(1):1-39.

35. Ljubkovic M, Gressette M, Bulat C, Cavar M, Bakovic D, Fabijanic D, Grkovic I, Lemaire C, Marinovic $\mathrm{J}$ : Disturbed Fatty Acid Oxidation, Endoplasmic Reticulum Stress, and Apoptosis in Left Ventricle of Patients With Type 2 Diabetes. Diabetes 2019, 68(10):1924-1933.

36. Sun J, Singh P, Osterlund J, Orho-Melander M, Melander O, Engstrom G, Edsfeldt A:

Hyperglycaemia-associated Caspase-3 predicts diabetes and coronary artery disease events. $J$ Intern Med 2021.

37. Pardali E, Sanchez-Duffhues G, Gomez-Puerto MC, Ten Dijke P: TGF-beta-Induced EndothelialMesenchymal Transition in Fibrotic Diseases. Int J Mol Sci 2017, 18(10).

38. Ma J, Sanchez-Duffhues G, Goumans MJ, Ten Dijke P: TGF-beta-Induced Endothelial to Mesenchymal Transition in Disease and Tissue Engineering. Front Cell Dev Biol 2020, 8:260.

39. Libby P, Theroux P: Pathophysiology of coronary artery disease. Circulation 2005, 111(25):34813488.

40. Bischoff J: Endothelial-to-Mesenchymal Transition. Circ Res 2019, 124(8):1163-1165.

41. Libby P: Inflammation in atherosclerosis. Nature 2002, 420(6917):868-874.

42. Rizzacasa B, Amati F, Romeo F, Novelli G, Mehta JL: Epigenetic Modification in Coronary Atherosclerosis: JACC Review Topic of the Week. J Am Coll Cardiol 2019, 74(10):1352-1365.

43. Westerterp M, Fotakis P, Ouimet M, Bochem AE, Zhang H, Molusky MM, Wang W, Abramowicz S, la Bastide-van Gemert S, Wang N et al: Cholesterol Efflux Pathways Suppress Inflammasome Activation, NETosis, and Atherogenesis. Circulation 2018, 138(9):898-912.

44. Niyonzima N, Bakke SS, Gregersen I, Holm S, Sandanger O, Orrem HL, Sporsheim B, Ryan L, Kong $\mathrm{XY}$, Dahl TB et al: Cholesterol crystals use complement to increase NLRP3 signaling pathways in coronary and carotid atherosclerosis. EBioMedicine 2020, 60:102985.

45. Martinez GJ, Celermajer DS, Patel S: The NLRP3 inflammasome and the emerging role of colchicine to inhibit atherosclerosis-associated inflammation. Atherosclerosis 2018, 269:262-271.

46. Jain T, Nikolopoulou EA, Xu Q, Qu A: Hypoxia inducible factor as a therapeutic target for atherosclerosis. Pharmacol Ther 2018, 183:22-33.

47. Roshan MH, Tambo A, Pace NP: The Role of TLR2, TLR4, and TLR9 in the Pathogenesis of Atherosclerosis. Int J Inflam 2016, 2016:1532832. 
48. Mitchell S, Vargas J, Hoffmann A: Signaling via the NFkappaB system. Wiley Interdiscip Rev Syst Biol Med 2016, 8(3):227-241.

49. Wang L, Wu H, Deng Y, Zhang S, Wei Q, Yang Q, Piao S, Bei W, Rong X, Guo J: FTZ Ameliorates Diabetic Cardiomyopathy by Inhibiting Inflammation and Cardiac Fibrosis in the Streptozotocin-Induced Model. Evid Based Complement Alternat Med 2021, 2021:5582567.

50. Wang C, Niimi M, Watanabe T, Wang Y, Liang J, Fan J: Treatment of atherosclerosis by traditional Chinese medicine: Questions and quandaries. Atherosclerosis 2018, 277:136-144.

51. Zhang J, Meng H, Zhang Y, Zhang X, Shao M, Li C, Tu P: The Therapeutical Effect of Chinese Medicine for the Treatment of Atherosclerotic Coronary Heart Disease. Curr Pharm Des 2017, 23(34):5086-5096.

52. Wang L, Xiang L, Piao S, Gong X, Zhou W, Feng W, Li H, Li L, Wei A, Zhu Q et al: The Efficacy and Safety of Chinese Medicine Fufang Zhenzhu Tiaozhi Capsule (FTZ) in the Treatment of Diabetic Coronary Heart Disease: Study Protocol for Multicenter, Randomized, Double-Blind, Placebo-Controlled Clinical Trial. Diabetes Metab Syndr Obes 2021, 14:2651-2659.

53. Chen Y, He X, Yuan X, Hong J, Bhat O, Li G, Li PL, Guo J: NLRP3 Inflammasome Formation and Activation in Nonalcoholic Steatohepatitis: Therapeutic Target for Antimetabolic Syndrome Remedy FTZ. Oxid Med Cell Longev 2018, 2018:2901871.

\section{Figures}


A

Ctrl

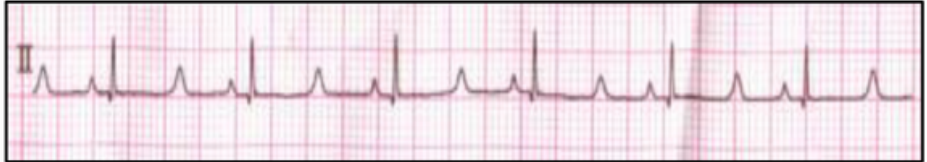

Mod

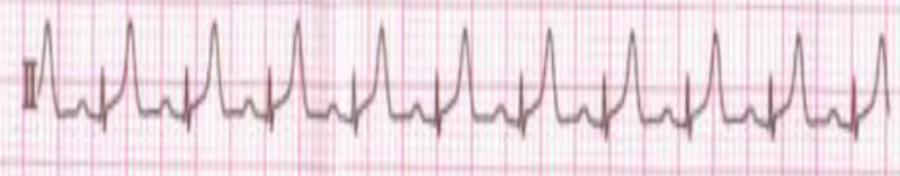

FTZ

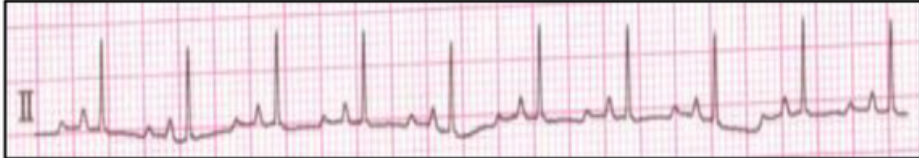

$\mathbf{M}+\mathbf{A}$

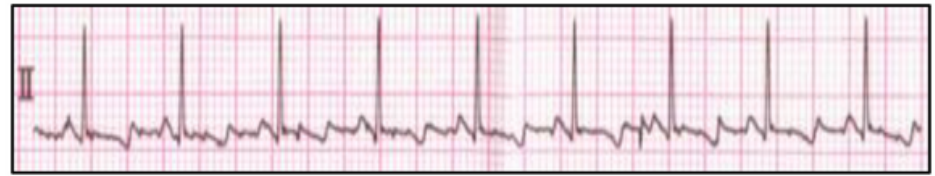

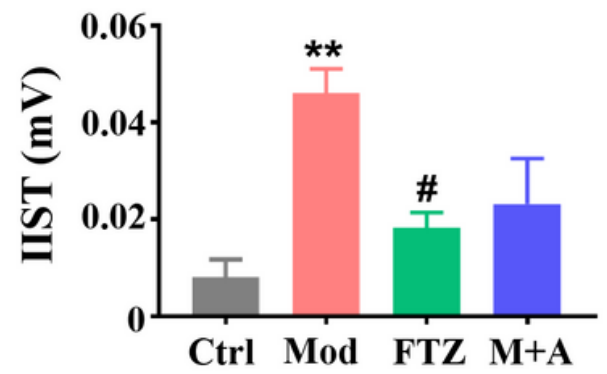

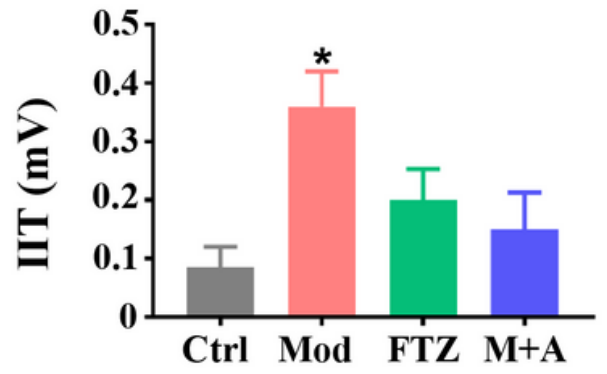

$\mathrm{B}$
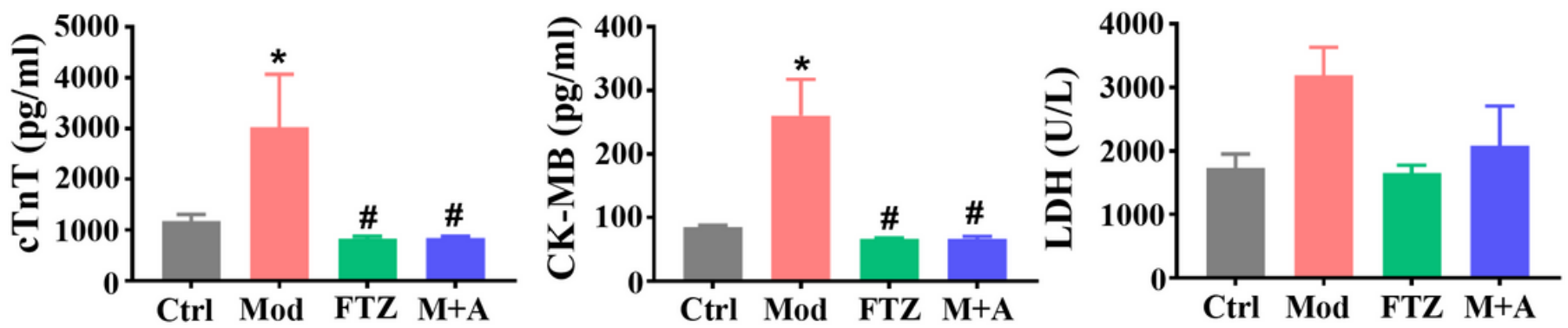

Figure 1

FTZ improves myocardial injury in minipigs. (A) The second lead of electrocardiogram was recorded in 12-lead ECG. (B) The serum levels of cardiac troponin T (CTnT), creatine kinase MB (CK-MB), and lactate dehydrogenase (LDH) in the Ctrl group, Mod group, and DM-CHD groups treated with FTZ or M + A. ${ }^{*} p<0.05,{ }^{*} p<0.01$, compared to Ctrl group; $\# p<0.05$, compared to Mod group (Ctrl, $n=4 ;$ Mod, $n=4 ; F T Z$, $n=5 ; M+A, n=5$ ) 

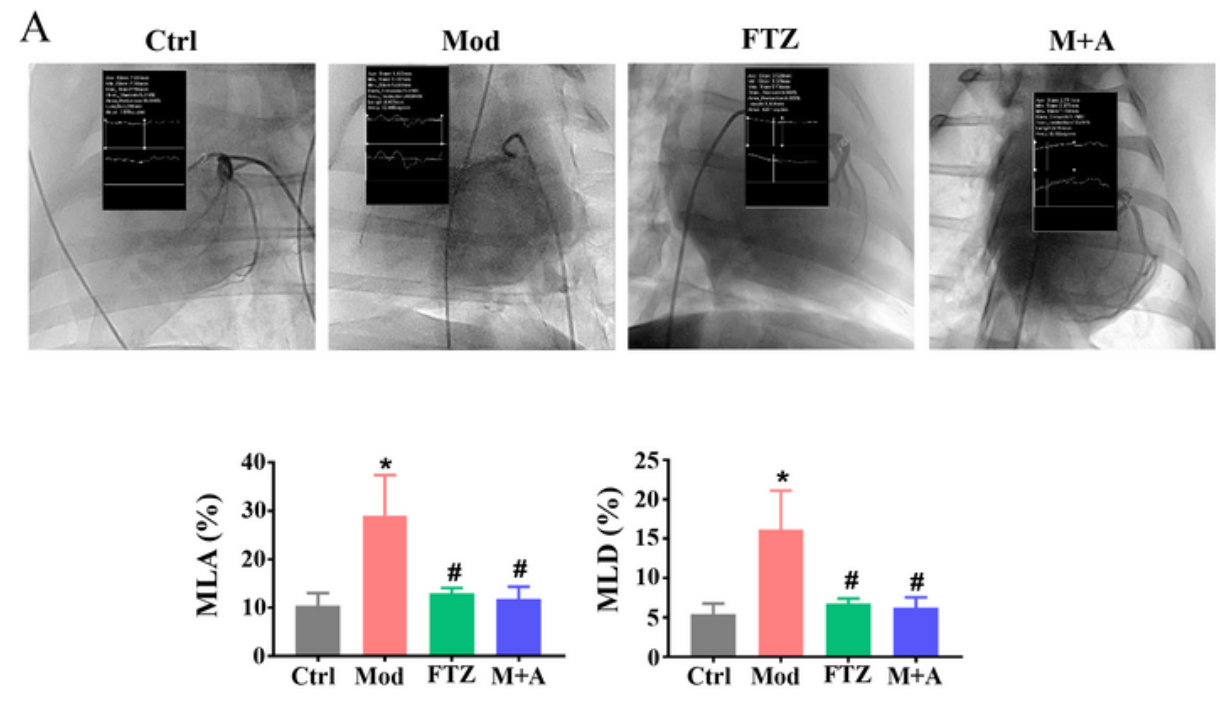

$\mathrm{B}$
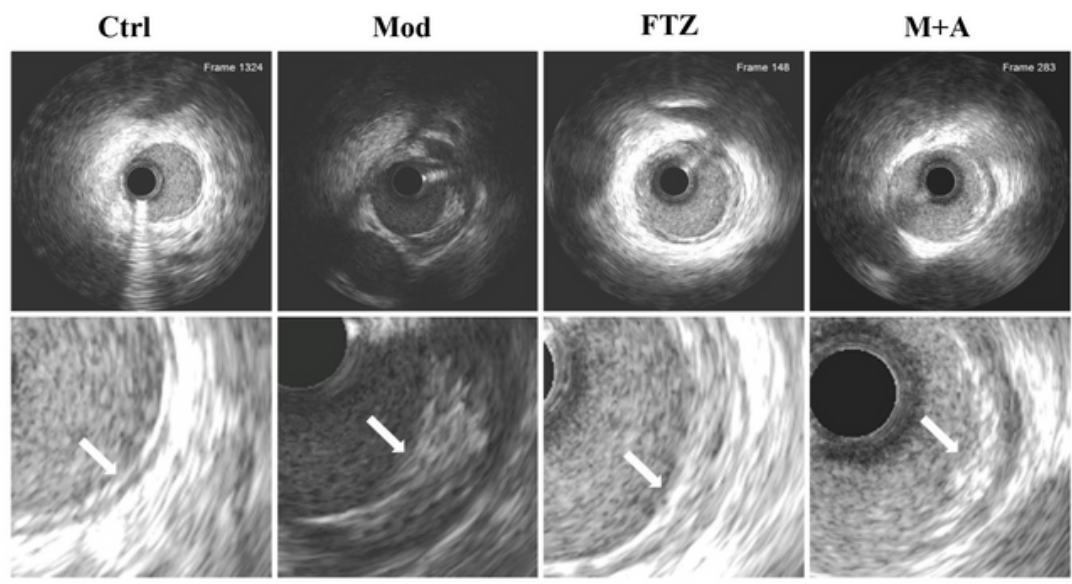

C

Ctrl


FTZ
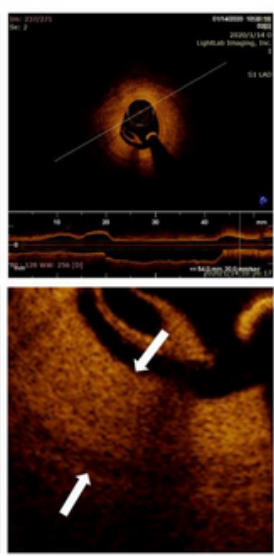
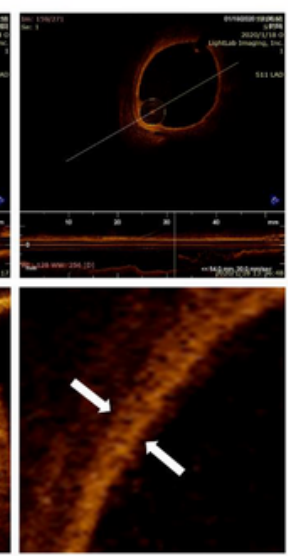

$\mathbf{M}+\mathbf{A}$
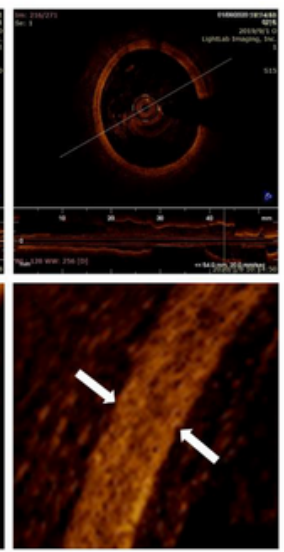

Figure 2

FTZ improved coronary stenosis in minipigs. (A) Angiography results and the changes of MLD\% and MLA\% of coronary artery in each group. (B) The results of IVUS (B) and OCT (C) in the Ctrl group, Mod group, and DM-CHD groups treated with FTZ or $\mathrm{M}+\mathrm{A}$. ${ }^{*} \mathrm{p}<0.05$, compared to Ctrl group; $\# \mathrm{p}<0.05$, compared to Mod group (Ctrl, $n=4 ; \operatorname{Mod}, n=4 ; F T Z, n=5 ; M+A, n=5$ ) 
A
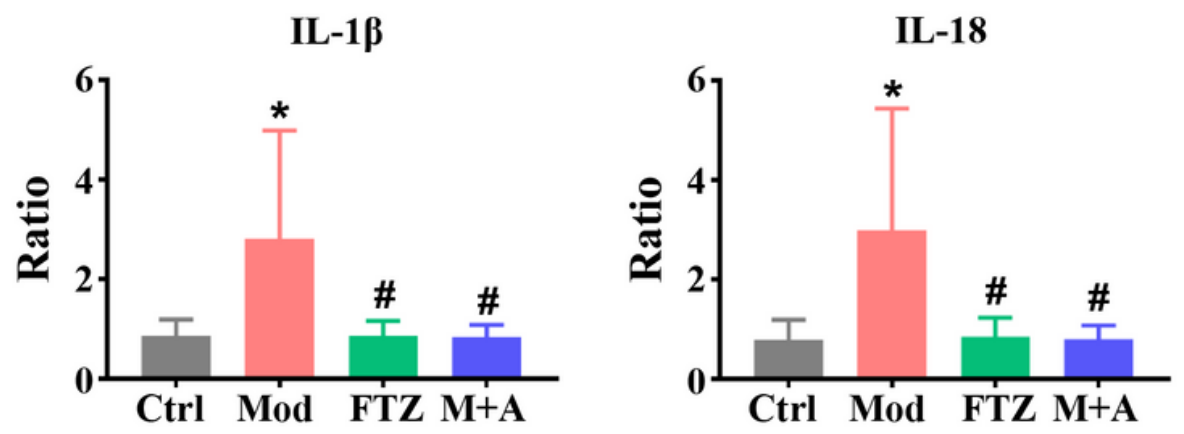

$\mathrm{B}$

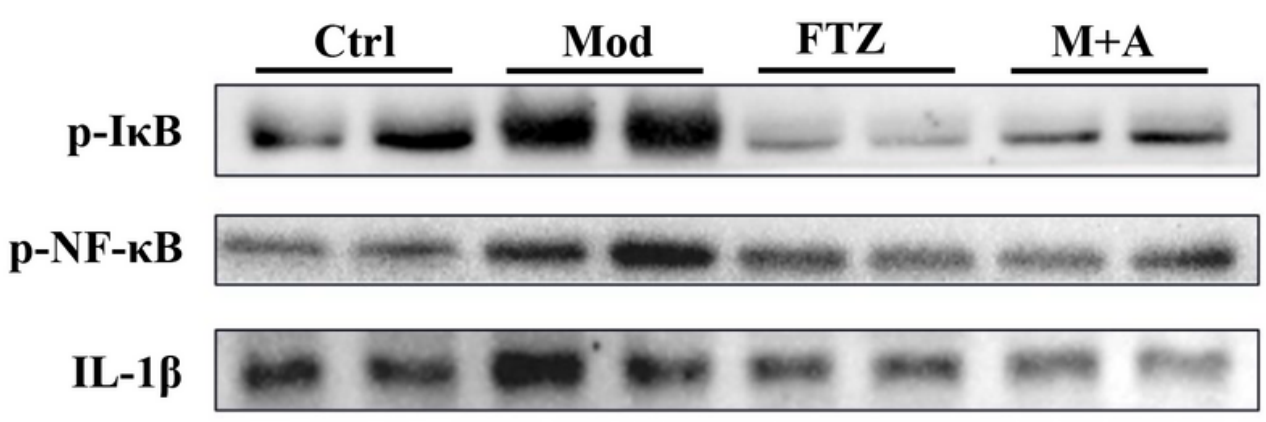

$\beta$-Tublin
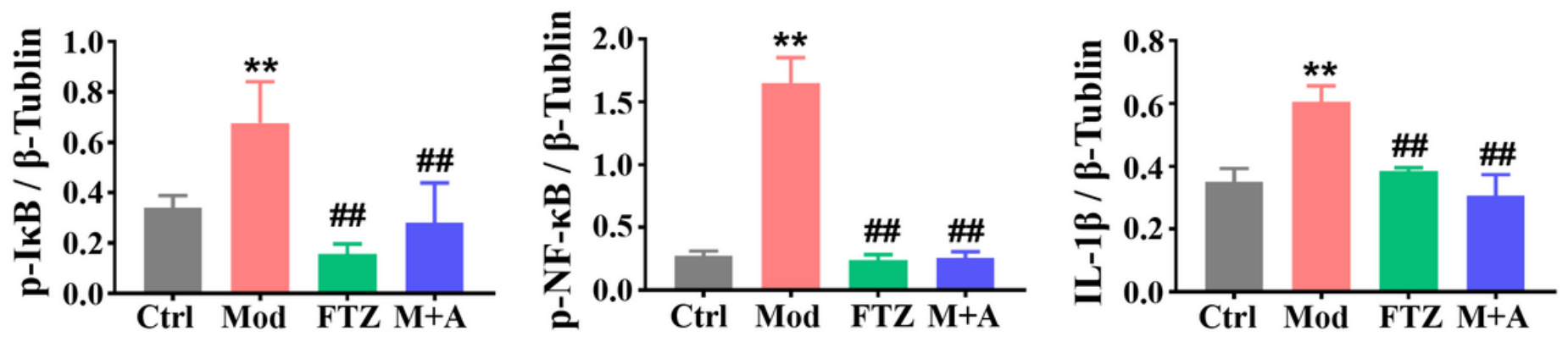

Figure 3

FTZ alleviated the inflammation in coronary artery of minipigs. (A) Changes in IL-1 $\beta$ and IL-18 in serum from beginning to end in each group. (B) The changes of phospho-IKB p40, phospho-NF-KB p65, and IL$1 \beta$ in the coronary artery tissues of different groups. ${ }^{*} p<0.05,{ }^{*} p<0.01$, compared to Ctrl group; $\# p<0.05$, $\# \# p<0.01$, compared to Mod group (Ctrl, $n=4 ; \operatorname{Mod}, n=4 ; F T Z, n=5 ; M+A, n=5)$ 

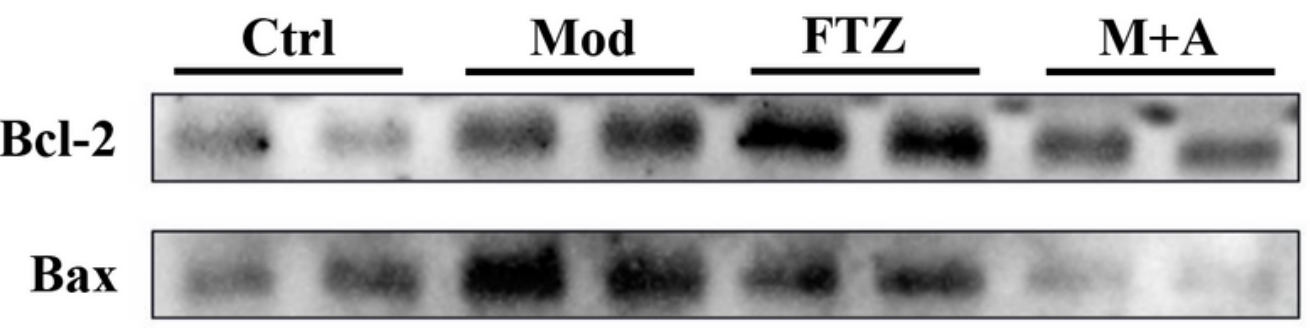

Cleave-

Caspase3

$\beta$-Tublin

$\mathrm{B}$
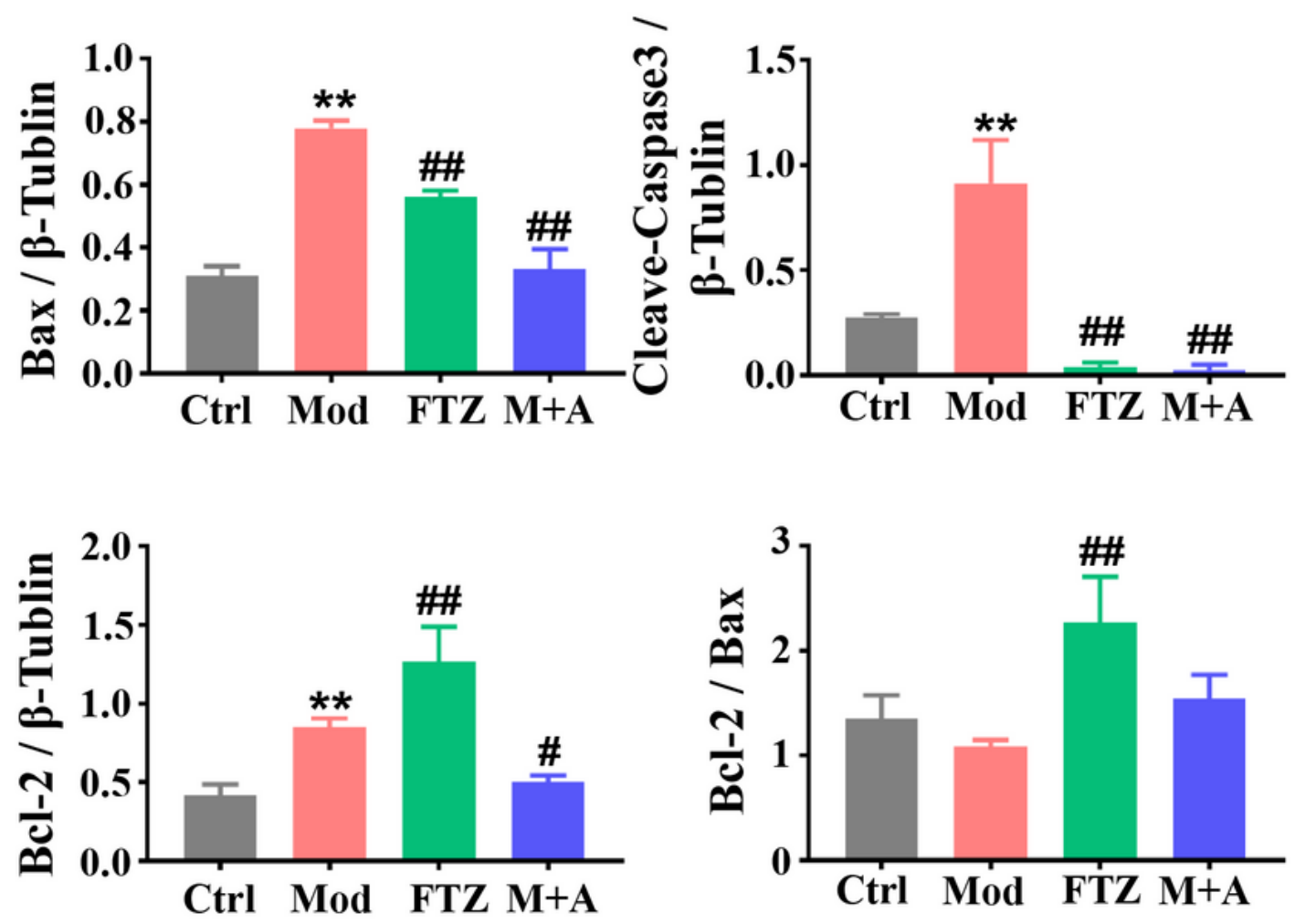

Figure 4

FTZ inhibited the apoptosis of coronary artery in minipigs. (A) The protein expression changes of Bcl-2, Bax, and cleave-Caspase 3 in the coronary artery tissues of different groups. (B) The quantitative analysis of $\mathrm{Bcl}-2$, Bax, and cleave-Caspase 3 proteins. ${ }^{*} \mathrm{p}<0.01$, compared to Ctrl group; $\# \mathrm{p}<0.05$, \#\#p<0.01, compared to Mod group (Ctrl, $n=4 ; \operatorname{Mod}, n=4 ; F T Z, n=5 ; M+A, n=5$ ) 
A
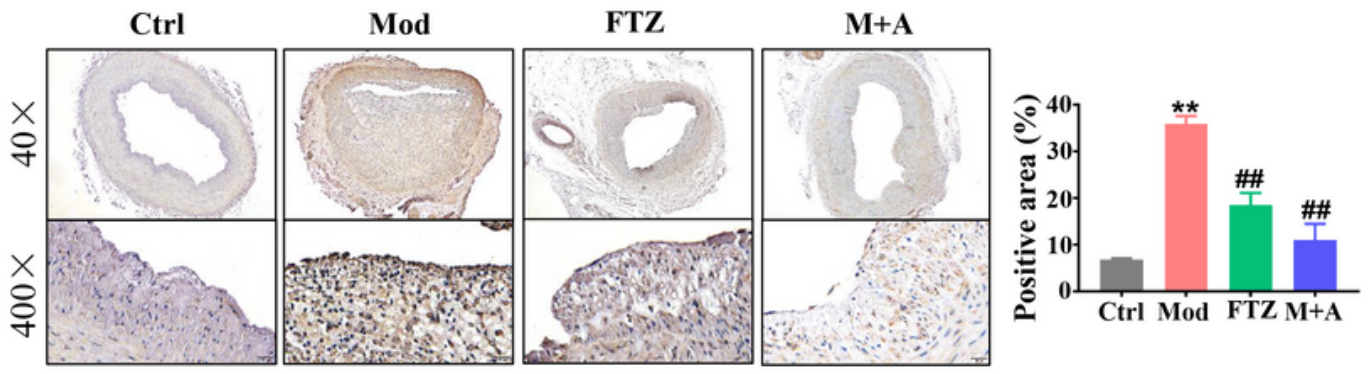

B
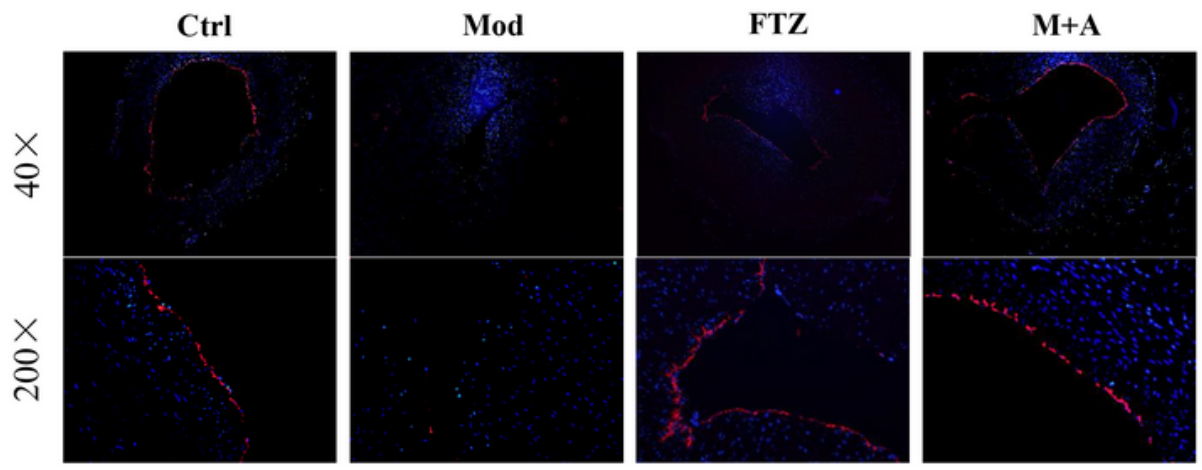

$\mathrm{C}$

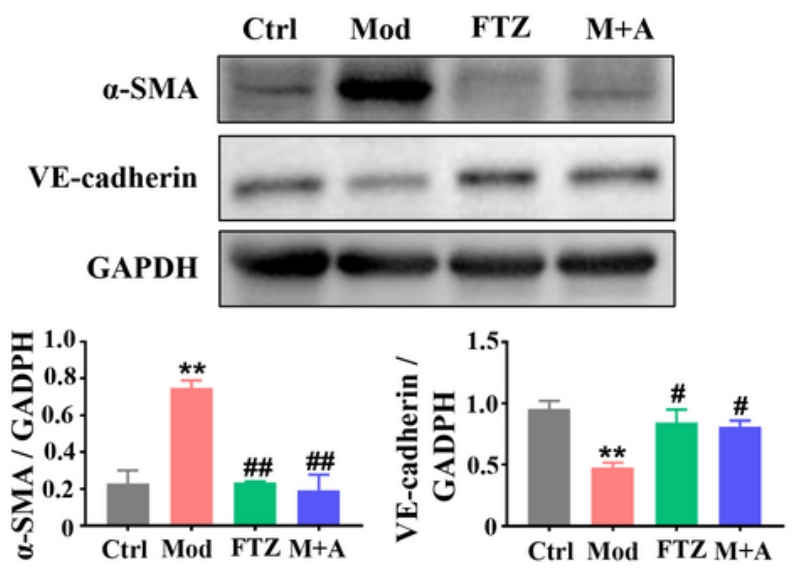

$\mathrm{D}$

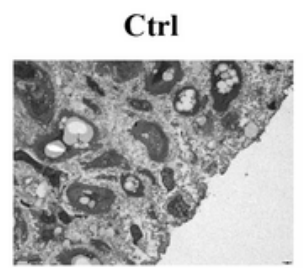

Mod

FTZ

$\mathbf{M}+\mathbf{A}$
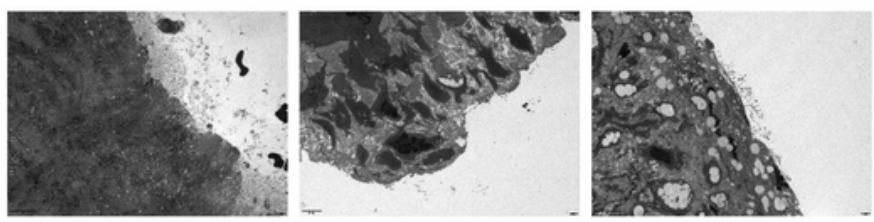

Figure 5

FTZ inhibited the coronary EndMT in DM-CHD minipigs. (A) The expression levels of a-SMA in the coronary artery of the minipigs were analyzed by immunohistochemistry. (B) The expression levels of CD31 in the coronary artery were analyzed by immunofluorescence staining. (C) The protein expressions and quantitative analysis of a-SMA and VE-cadherin in the coronary artery tissues of different groups. (D) The results of electron microscopic study of coronary artery vascular endothelium. ${ }^{*} p<0.05,{ }^{*} p<0.01$, 
compared to Ctrl group; \#p<0.05, \#\#p<0.01, compared to Mod group (Ctrl, $n=4 ;$ Mod, $n=4 ; F T Z, n=5$; $M+A, n=5)$

A

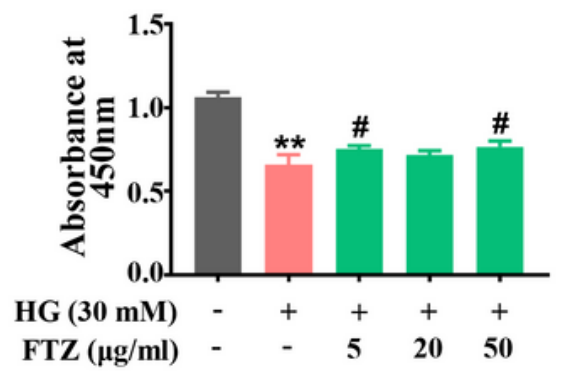

$\mathrm{C}$

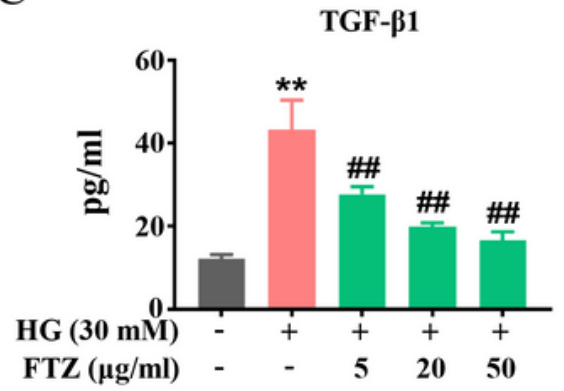

B

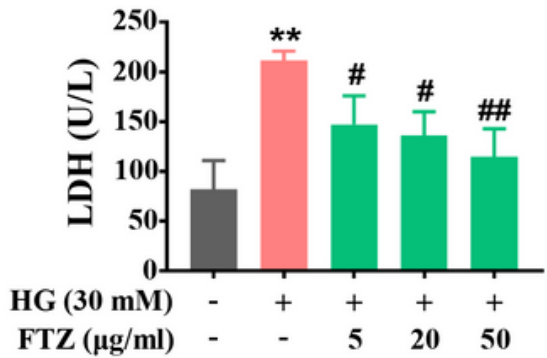

$\mathrm{D}$

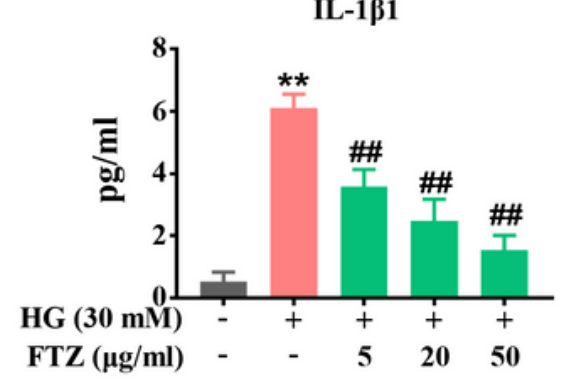

$\mathrm{E}$

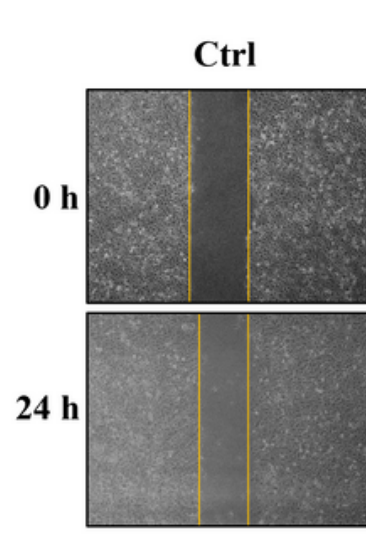

HG+FTZ

HG+FTZ

$(5 \mu \mathrm{g} / \mathrm{ml})$

(20 $\mu \mathrm{g} / \mathrm{ml})$

HG+FTZ
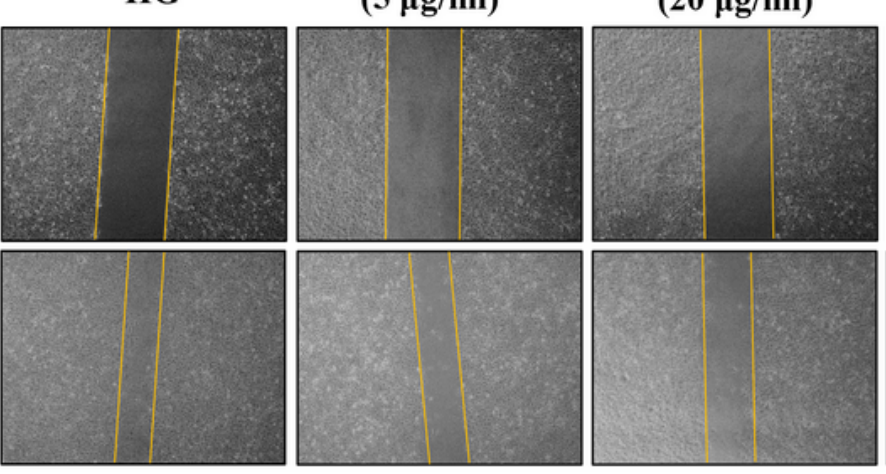

(50 $\mu \mathrm{g} / \mathrm{ml})$
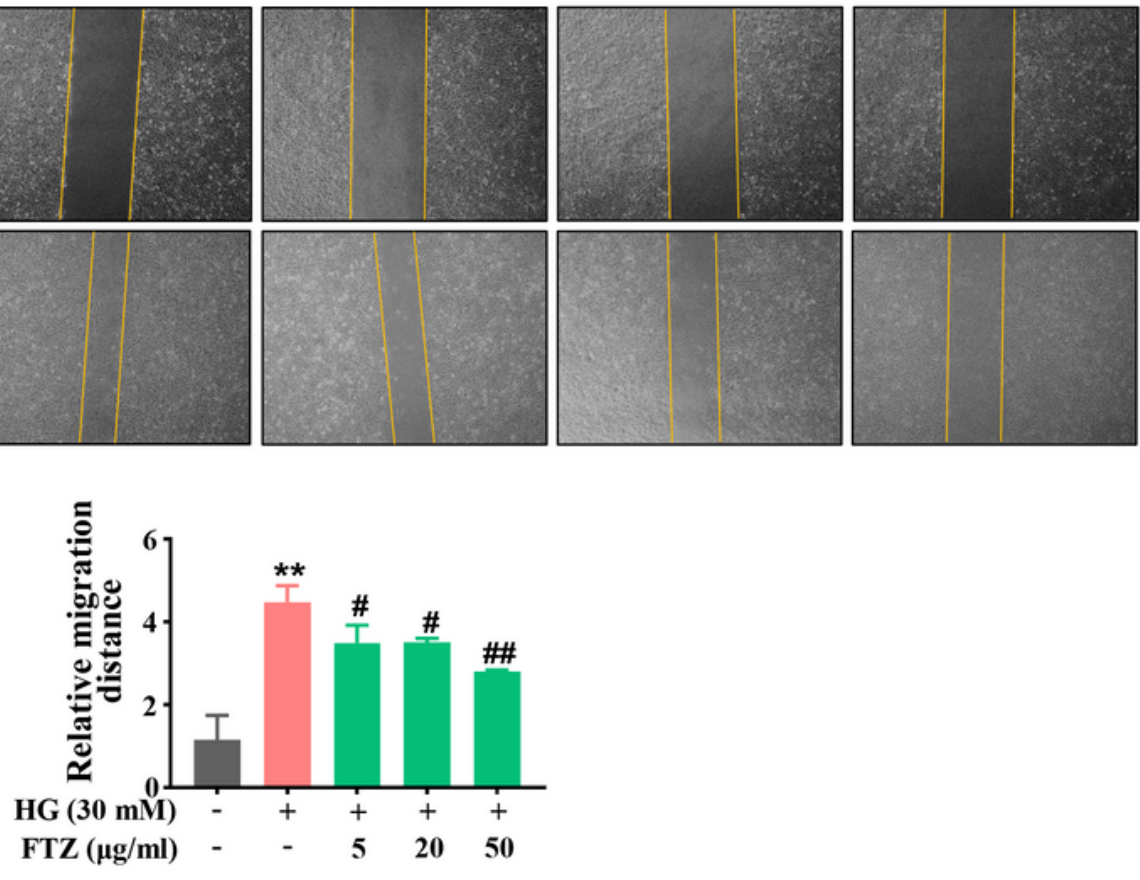

Figure 6

FTZ ameliorated the effect of high glucose on HUVECs. HUVECs were treated with high glucose (HG, 30 $\mathrm{mM}$ ) and different concentrations of FTZ for $24 \mathrm{~h}$ or $48 \mathrm{~h}$. Cell viability was analyzed by CCK-8 assay (A). The levels of LDH (B), TGF- $\beta 1(C)$, and IL-1 $\beta$ (D) in supernatant were tested by ELISA. The migration 
activity of HUVECs was tested by cell scratch assay (E). ${ }^{* *} \mathrm{p}<0.01$, compared to Ctrl (HG-FTZ-); \#p<0.05, $\#$ \# $<0.01$, compared to $H G(n=3)$

\section{Supplementary Files}

This is a list of supplementary files associated with this preprint. Click to download.

- Supplementarymaterials.docx

- sFigure1.tif

- sFigure2.tif

- sFigure3.tif 